\title{
Scaling Properties of Dynamical Localization in Monochromatically Perturbed Quantum Maps: standard map and Anderson map
}

\author{
Hiroaki S. Yamada \\ Yamada Physics Research Laboratory, Aoyama 5-7-14-205, Niigata 950-2002, Japan \\ Fumihiro Matsui \\ Department of Physics, College of Science and Engineering, \\ Ritsumeikan University Noji-higashi 1-1-1, Kusatsu 525-8577, Japan \\ Kensuke S. Ikeda \\ College of Science and Engineering, Ritsumeikan University Noji-higashi 1-1-1, Kusatsu 525-8577, Japan
}

(Dated: October 11, 2018)

\begin{abstract}
Dynamical localization phenomena of monochromatically perturbed standard map (SM) and Anderson map (AM), which are both identified with a two-dimensional disordered system under suitable conditions, are investigated by the numerical wavepacket propagation. Some phenomenological formula of the dynamical localization length valid for wide range of control parameters are proposed for both SM and AM. For SM the formula completely agree with the experimentally established formula, and for AM the presence of a new regime of localization is confirmed. These formula can be derived by the self-consistent mean-field theory of Anderson localization on the basis of a new hypothesis for cut-off length. Transient diffusion in the large limit of the localization length is also discussed.

PACS numbers: 05.45.Mt,71.23.An,72.20.Ee
\end{abstract}

\section{INTRODUCTION}

In one-dimensional quantum systems, strong localization phenomena have been observed due to large quantum interference effect when disorder exists in the system. A quite similar localization phenomenon occurs in classically chaotic dynamical systems which exhibit chaotic diffusion in the classical limit. A typical example of the former is one-dimensional disordered systems (1DDS) 1, 2], and the latter one is the quantum standard map (SM) 3]. It has been shown that the localization of the wavepacket can be delocalized by applying dynamical perturbation composed of a few number of coherent modes [4 11]. If the number of the modes is more than two, the delocalization takes place through a localizationdelocalization transition (LDT) accompanied by remarkable critical phenomena as the perturbation strength is increased. It has been explored in detail the transition process from the localized phase for mode number more than two.

In the previous paper 12], we also investigated quantum diffusion of an initially localized wavepacket in the polychromatically perturbed Anderson map (AM) which is a time-discretized version of the Anderson model, in comparison with the SM driven by the same polychromatic perturbation. However, the nature of quantum diffusion exhibited by the monochromatically perturbed AM and SM, which has been supposed to be localized, have not still been well-investigated, except for early stage studies on SM [13].

Experimentally, Manai et al. observed the critical phenomenon of the LDT for Cesium atoms in optical lattice [6], which is an experimental implementation of the per- turbed SM, and the observed results were successfully interpreted as a three-dimensional LDT based on the equivalence between SM and multi-dimensional disordered lattice by the so-called Maryland transform [14]. Their results are also interpreted by the self-consistent theory (SCT) of the weak localization in three dimensional disordered system (3DDS) [4 11]. Further, they recently observed the localization phenomenon in the SM driven by coherent monochromatic perturbation [15]. This work is a very important experimental contribution in the sense that it first succeeded in realizing the two-dimensional disordered system (2DDS) as a monochromatically perturbed SM in the optical lattice. In order to confirm the presence of localization, very long time-scale data must be examined, which is very difficult in real experiment but is much easier in numerical simulation. After the early report suggesting the presence of localization [13], there has been no work of numerical simulation for the monochromatically perturbed SM. The experimental results should be examined by reliable numerical simulation taking sufficiently long time steps, which will be done in the present paper. We note also that there have been several studies on the localization of the copuled SM, which can be identified with the 2DDS by the Maryland transformation [16, 17], but no definite quantitative results has been estabilished, because long time-scale simulation of coupled rotors is much more difficult than the monochromatically perturbed SM.

There are two main purposes in the present paper. One is to explore systematically the localization characteristics of the monochromatically perturbed SM with the numerical and theoretical methods. We focus our investigation on the quantum regime in which the coupling 
strength is smaller than a characteristic value decided by the Planck constant, and reexamine the validity of the experimentally observed result of the Manai et al. in a wide parameter range of the quantum regime. Our results are interpreted by the SCT of the localization based on a newly proposed hypothesis.

Another purpose is to report the characteristics of monotonically perturbed AM in comparison with the perturbed SM mentioned above, whose localization property has not been fully investigated. The AM is close to the original model of random lattice proposed by Anderson in a sense that randomness is explicitly included, and has its own physical origin quite different from the SM. Note that there have already been some publications for numerical results of AM [18 20], and the presence of localization phenomenon for unperturbed and monochromatically perturbed AM was stressed, but the present paper is the first detailed quantitative exploration of the dynamical localization length and the scaling properties for the monochromatically perturbed AM. The parameter dependence of the localization length on the disorder strength and perturbation strength are given numerically, and it is theoretically interpreted based on the SCT of the localization.

Our main concern is whether or not the above mentioned two models with quite different physical origin share common features of the dynamical localization phenomenon. The outline of the paper is as follows. In the next section, we introduce model systems, monochromatically perturbed SM and AM, examined in the present paper. The numerical results of scaling properties of the localization length in the perturbed SM and AM are given in Sects. III and IV respectively, and some empirical formula representing the localization characteristic are proposed. In particular, the existence of two different regimes of the localization is confirmed for AM. In Sect. V] these formula are consistently derived by the SCT of Anderson localization for anisotropic 2DDS [21, 22] by introducing some hypothesis for the characteristic length as a cut-off in the self-consistent equation. In addition, the relation between SM and AM is made clear by the Maryland transform [14]. In Sect. VI] we discuss the characteristics of diffusion for both models observed transiently on the way to the final localization. The existence of the semiclassical regime beyond the quantum regime is emphasized for SM. The last section is devoted to summary and discussion. In appendixes, we give some complementary numerical results and a simple derivation of the Maryland transform in Sect. V.

\section{MODELS}

The model Hamiltonian of the periodically kicked system driven by dynamical perturbation with different frequency $\omega_{1}$ from those of the kick is

$$
H(\hat{p}, \hat{q}, t)=T(\hat{p})+V(\hat{q})\left\{1+\epsilon \cos \left(\omega_{1} t+\varphi_{0}\right)\right\} \delta_{t},
$$

where

$$
\delta_{t}=\sum_{m=-\infty}^{\infty} \delta(t-m \tau)=\frac{1}{\tau} \sum_{m=-\infty}^{\infty} \cos \left(\frac{2 \pi}{\tau} m t\right) .
$$

Thus the system is kicked by the periodic delta-functional force with the period $\tau$. Here $T(\hat{p})$ and $V(\hat{q})$ represent translational kinetic energy and potential energy, respectively. And $\hat{p}$ and $\hat{q}$ are momentum and positional operators of the kicked system, respectively. The evolution for the single step between the time interval $[s \tau,(s+1) \tau]$ is represented by the unitary operator

$$
U\left(s \tau, \varphi_{0}\right)=\mathrm{e}^{-i T(\hat{p}) \tau / \hbar} \mathrm{e}^{-i V(\hat{q})\left\{1+\epsilon \cos \left(\omega_{1} \tau s+\varphi_{0}\right)\right\} / \hbar},
$$

which depends explicitly upon the step $s \in \mathbb{Z}$. The sinusoidal periodic perturbation is characterized by the frequency $\omega_{1}$ incommensurate with the kick frequency $2 \pi / \tau$ and the strength $\epsilon$. We can take the effect of the periodic force into account by introducing an additional linear oscillator $\omega_{1} \hat{J}$ acting as the periodic force to the kicked system, which we call $J$-oscillator hereafter. $\varphi_{0}$ is an initial phase of the oscillation. Then, instead of the Hamiltonian of Eq.(11), we consider the Hamiltonian of two degrees of freedom

$$
H_{t o t}(\hat{p}, \hat{q}, \hat{J}, \hat{\phi}, t)=T(\hat{p})+\omega_{1} \hat{J}+V(\hat{q})(1+\epsilon \cos \hat{\phi}) \delta_{t},(4)
$$

where $\hat{\phi}=\phi, \hat{J}=-i \hbar d / d \phi$ are the angle-action operators in the angle representation, and the angle variable is defined in the section $[0,2 \pi]$. The corresponding unitary evolution operator for the kick period $\tau$ does no longer depends upon the step $s$, and is an "autonomous" evolution operator

$$
U_{t o t}=\mathrm{e}^{-i \omega_{1} \hat{J} \tau / \hbar} \mathrm{e}^{-i T(\hat{p}) \tau / \hbar} \mathrm{e}^{-i V(\hat{q})(1+\epsilon \cos \hat{\phi}) / \hbar} .
$$

One can show the relation between the autonomous evolution operator Eq.(5) and the non-autonomous evolution operator of Eq.(31) as

$$
\begin{aligned}
U_{\text {tot }}^{s} & =\mathcal{T} \mathrm{e}^{-i \int_{0}^{s} H_{\text {tot }}\left(s^{\prime}\right) d s^{\prime} / \hbar} \\
& =\mathrm{e}^{-i \omega_{1} J \tau s / \hbar} \mathcal{T} \mathrm{e}^{-i \int_{0}^{s} d t^{\prime}\left[T(\hat{p}) \tau+V(\hat{q})\left\{1+\epsilon \cos \left(\omega_{1} t^{\prime}+\varphi_{0}\right)\right\}\right] / \hbar} \\
& =\mathrm{e}^{-i \omega_{1} J \tau s / \hbar} U\left(s \tau, \varphi_{0}\right) U\left((s-1) \tau, \varphi_{0}\right) \ldots U\left(\varphi_{0}\right) .
\end{aligned}
$$

where $\mathcal{T}$ is the time ordering operator. Suppose that we take the action eigenstate, for example, $|J=0\rangle$ as the initial state of the $J$-oscillator. It is represented by the Fourier sum over the angle eigenstates as $|J=0\rangle=\frac{1}{\sqrt{J}} \sum_{j}^{J}\left|\phi_{j}\right\rangle$, where $\phi_{j}=2 \pi j / J$. Then, applying Eq.(3), the wavepacket propagation by $U_{\text {tot }}$ launched from the state $|J=0\rangle \otimes\left|\Psi_{0}\right\rangle$, where $\left|\Psi_{0}\right\rangle$ is an initial state of the kicked oscillator, is achieved by applying the periodically perturbed evolution operator $U\left(t \tau, \varphi_{0}\right)$ of Eq.(3) to the initial state $\left|\phi_{0}\right\rangle \otimes\left|\Psi_{0}\right\rangle$ and next summing over $\phi_{0}$. Summation over $\varphi_{0}$ can be replaced very well by the ensemble average over randomly chosen $\varphi_{0}[23]$. We can thus use the representation of Eq.(3) for the numerical 
wavepacket propagation. But in theoretical considerations we often return to the autonomous representation of Eq. (15).

In the present paper we set $T(\hat{p})=\hat{p}^{2} / 2, V(\hat{q})=$ $K \cos \hat{q}$ for SM, and $T(\hat{p})=2 \cos (\hat{p} / \hbar)=\left(\mathrm{e}^{\partial / \partial q}+\mathrm{e}^{-\partial / \partial q}\right)$ (hopping between nearest neighbour sites), $V(\hat{q})=$ $W v(\hat{q})=W \sum_{n} \delta(q-n) v_{q}|q\rangle\langle q|$ for AM, respectively, where on-site potential $v_{n}$ takes random value uniformly distributed over the range $[-1,1]$ and $W$ denotes the disorder strength.

In the autonomous representation, the Heisenberg equation (classical equation) of motion describing the monochromatically perturbed SM is

$$
\left\{\begin{array}{l}
p_{s+1}-p_{s}=K \sin q_{s}\left(1+\epsilon \cos \phi_{s}\right) \\
q_{s+1}-q_{s}=p_{s+1} \tau \\
J_{s+1}-J_{s}=K \epsilon \cos q_{s} \sin \phi_{s} \\
\phi_{s+1}-\phi_{s}=\omega_{1} \tau
\end{array}\right.
$$

where the Heisenberg operator is defined by $X_{s} \equiv$ $U^{-s} X U^{s}$. The set of equation for the monochromatically perturbed AM can be also obtained formally by the same way, but we should note that they have no the classical counterpart.

Let us consider the relation between the discretized system and the time-continuous system using unperturbed cases $(\epsilon=0)$ for simplicity. We take the symmetrized unit of time section $[s \tau-\tau / 2, s \tau+\tau / 2]$ instead of $[s \tau,(s+1) \tau]$, then we have the symmetrized form of the single step evolution operator $U_{S Y}=$ $\mathrm{e}^{-i \tau T / 2 \hbar} \mathrm{e}^{-i V / \hbar} \mathrm{e}^{-i \tau T / 2 \hbar}$ instead of Eq.(3). It is wellknown that $U_{S Y}$ approximates $\mathrm{e}^{-i \tau(T+V / \tau) / \hbar}$ up to the correction of $O\left((\tau / \hbar)^{3}\right)$, which is the lowest order BakerHausdorff-Campbell expansion of incommutable operator product. Hence, if we make $\tau / \hbar \ll 1$ keeping $V / \tau \sim O(1)$, the time evolution by $U_{S Y}$ is closely approximated by that of the time-continuous Hamiltonian $T+V / \tau$, and the AM agrees with the Anderson model of 1DDS, whereas the SM becomes the gravitational pendulum. (The condition $\tau / \hbar \ll 1$ is relaxed to $\tau \ll 1$ if the system has the classical limit.) We hereafter choose the period as $\tau=1$ throughout the present paper.

We explain here the numerical wavepacket propagation based upon the nonautonomous unitary evolution operator Eq. (3) in which $\varphi$ is taken as classical random number. The localization phenomena take place in the momentum $(p)$ space for SM and in the position $(q)$ space for AM, respectively. The momentum space of SM and the position space of AM are spanned by the momentum or position eigenfunctions commonly denoted by $|n\rangle$, where $n \in \mathbb{Z}$, and the momentum and position are quantized as $p=n \hbar$ for SM and $q=n$ for AM. Further the periodic boundary condition is imposed on the wavefunction $|\Psi\rangle$ as $\langle n+N \mid \Psi\rangle=\langle n \mid \Psi\rangle$ for momentum (SM) and position (AM) representations, and so the integer value $n$ is bounded as $-N / 2 \leq n \leq N / 2$ for a very large positive integer $N$.

Let the wavepacket at the time $t$ be

$$
\left|\Psi_{s}\right\rangle=U\left(s, \varphi_{0}\right) U\left((s-1), \varphi_{0}\right) \ldots U\left(\varphi_{0}\right)\left|\Psi_{0}\right\rangle
$$

starting with the localized state $\left|\Psi_{0}\right\rangle=\left|n_{0}\right\rangle$ in the momentum (SM) or in the position (AM) space. We monitor the time-dependent mean square displacement (MSD), $m_{2}(t)=\left\langle\sum_{n=-\infty}^{\infty}\left(n-n_{0}\right)^{2}|u(n, t)|^{2}\right\rangle_{\Omega}$ for the propagating wavepacket $u(n, t)=\left\langle n \mid \Psi_{t}\right\rangle$, where $\langle\ldots\rangle_{\Omega}$ denotes the ensemble average over initial condition $n_{0}$ for SM and different random configuration of $v(n)$ for AM, respectively. In addition, the average over $\varphi_{0}$ should be taken, but the $\varphi_{0}$ dependence of the MSD is much weaker compared with the dependency upon $n_{0}$ (for SM) and sample of $v(n)$ (for AM), and the averaging is ignored if unnecessary.

In this paper, we compute the localization length (LL) of the dynamical localization, $p_{\xi}=\sqrt{m_{2}(\infty)}$ for SM and $\xi=\sqrt{m_{2}(\infty)}$ for AM, after numerically calculating the MSD for long-time, where $m_{2}(\infty)$ is numerically saturated MSD. In fact, Fig. [1]shows the time-dependence of MSD for some unperturbed and perturbed cases in SM and AM. It is found that the growth of time-dependence is saturated and the LL becomes larger values as the perturbation strength becomes larger.

Note that it is difficult to get the accurate LL as the perturbation strength increases for cases with the larger $K / \hbar$ in SM and smaller $W$ in AM because of explosive increase of MSD. Then we also use the time-dependent diffusion coefficients to characterize the transient behaviour before reaching the LL as will be discussed in Sect VI.

\section{LOCALIZATION PHENOMENA IN MONOCHROMATICALLY PERTURBED QUANTUM SM}

In this section we show the localization characteristics of the monochromatically perturbed SM with changing the three parameters $K, \hbar$ and $\epsilon$ in a wide range.

As was already demonstrated in the experiments by the Manai et al., a remarkable feature of the SM with monochromatic perturbation is a definite exponential growth of the localization length with respect to the perturbation strength $\epsilon$ [15], namely,

$$
p_{\xi}=D \exp \{\epsilon A\},
$$

where the constants $A, D$ are determined by $K$ and $\hbar$. The experiment of the Manai et al. was done for $\hbar$ greater than unity, i.e. in a strong quantum regime. On the other hand, it has been numerically and experimentally observed that, if $\hbar$ is small enough, classical diffusion of coupled SMs, which can be identified with 2DDS, is restored over a long time scale. Problems related to the classical diffusion will be discussed in SectVI.

In this section, we focus on the results of numerical experiments in the "quantum regime" where $\epsilon$ is smaller than a certain characteristic value dependent upon $\hbar$, which will be discussed later.

Figure2 2shows $\epsilon$-dependence of the localization length $p_{\xi}$ for some $\hbar$ 's and $K$ 's. All the plots tell that the expression of (9) works quite well. Validity of Eq.(9) 

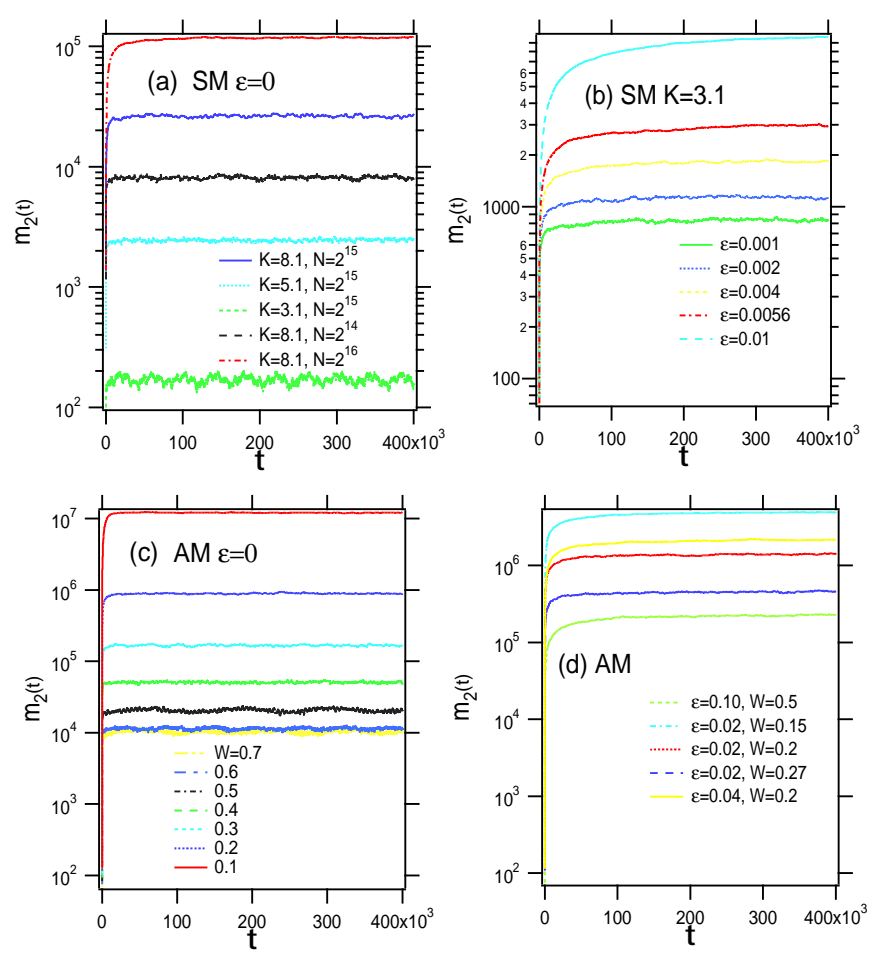

FIG. 1: (Color online) The time-dependence of the MSD of SM and AM. (a)Unperturbed SM for $K=3.1,5.1,8.1$ with $\hbar=\frac{2 \pi 1248}{2^{15}}$, and for $K=8.1$ with $\hbar=\frac{2 \pi 1248}{2^{14}}, \frac{2 \pi 1248}{2^{16}}$. (b)Monochromatically perturbed SM for $K=3.1$ and $\hbar=$ 0.12 with $\epsilon=0.0001 \sim 0.1$ from below. (c) Unperturbed AM with $W=0.1 \sim 0.6$ from below. (d)Monochromatically perturbed AM with some combinations of $\epsilon$ and $W$. Note that the horizontal axes are in logarithmic scale. The system and ensemble sizes are $N=2^{15}-2^{16}$ and $10-50$, respectively, thorough this paper.

was confirmed for all values of $K$ and $\hbar$ we examined. In the following, we discuss the $K$-dependence and the $\hbar$-dependence of the coefficients $A, D$, by the intercept and the slope numerically determined by the semi-log plot of Fig 2 .

First, we show the variation of the coefficient $A$ and coefficient $D$ for the change of $K$ with fixing the ratio $K / \hbar \equiv \kappa$. It is shown in Fig](a) for the three values $\kappa=\kappa_{0}, \kappa_{0} / 2, \kappa_{0} / 4$, where $\kappa_{0}=18$. Obviously, it turns out that the variation in the value of the coefficient $A$ maintains a nearly constant value when the ratio $\kappa$ is constant if some irregular variation is ignored. These facts means that $A$ and $D$, which is a function of $\kappa$ and $K$, should be the function of $\kappa$ alone. Next, we change $\hbar$ for various fixed values of $K$, which is shown in Fig 4 (a). Apparently, for all values of $K$ the dependence $A \propto \hbar^{-2}$ is observed. Since $A$ depends only on $\kappa$, the following relation should hold

$$
A=\text { const. }\left(\frac{K}{\hbar}\right)^{2} \propto \kappa^{2} .
$$
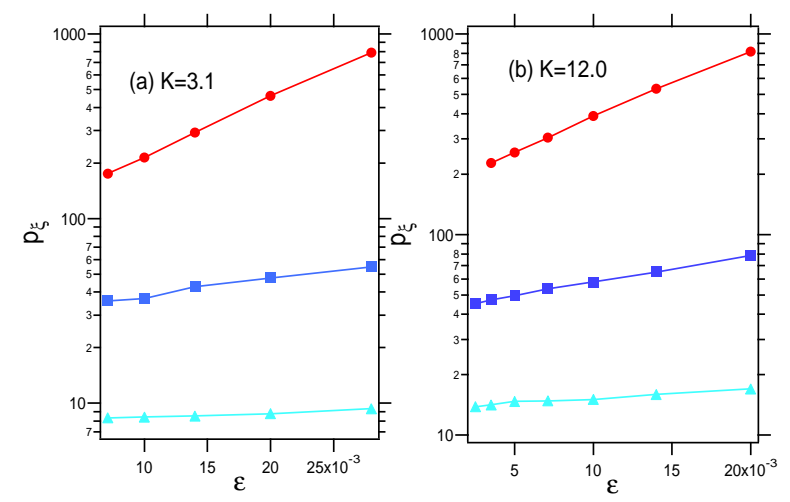

FIG. 2: (Color online) Localization length $p_{\xi}$ as a function of the relatively small perturbation strength $\epsilon$ in the quantum regime $\left(\epsilon=1 \times 10^{-3} \sim 30 \times 10^{-3}\right)$. (a) $K=3.1$ for $\hbar=\frac{2 \pi 435}{2^{12}}, \frac{2 \pi 435}{2^{13}}, \frac{2 \pi 435}{2^{14}}$ from below. (b) $K=12.0$ for $\frac{2 \pi 1741}{2^{12}}$, $\frac{2 \pi 1741}{2^{13}}, \frac{2 \pi 1741}{2^{14}}$ from below. Note that the horizontal axes are in logarithmic scale.

Actually, the prediction is confirmed by the fact that the scaled coefficient $A / \kappa^{2}$ is almost constant for different $K$ and $\kappa$ (and so $K$ and $\hbar$ ) as is shown in Fig 3. (c).

On the other hand, the $(K / \hbar)$-dependence of another coefficient $D$ is shown in Fig 3 (b) and Fig鸟(b). It is expected that the coefficients $D$ and $A$ should show a similar behaviour except for numerical prefactors, i.e. $D \sim A$. However, the $K$-dependence of the coefficient $D$ is less definite and it is often accompanied by some irregular fluctuations. This fluctuation becomes more pronounced as the $\kappa$ is larger, in other words, the smaller $\hbar$ enhances fluctuation, as recognized from Fig 4(b). This phenomenon is caused by the so-called acceleration modes which are peculiar to the classical dynamics of SM. Actually, in the values of $K=2 n \pi$ where the classical acceleration mode exists, the classical diffusion coefficient $D_{c l}$ increases explosively, and also reflects the localization length of the quantum system as shown in the peak around $K=7$. Increasing perturbation strength $\epsilon$ reduces the effect of acceleration mode. The acceleration modes existing with zero measure in the classical system plays very complicated roles, but it is not essential to the discussion of the quantum localization phenomenon, so we will not discuss it in detail in this paper. If we ignore such a fluctuation, the dependence of the coefficient $D$ on $K$ and $\hbar$ in Fig 3 (b) and Fig 4(b) exhibits very similar behaviour to the coefficient $A$ and so we conclude that

$$
D=\text { const. }\left(\frac{K}{\hbar}\right)^{2} \propto \kappa^{2} .
$$

This prediction is confirmed also by the plot of the scaled coefficient $D / \kappa^{2}$ in FigB(d) in a way parallel to Fig B(c).

To summarize the facts presented so far, localization length of monochromatically perturbed SM is repre- 

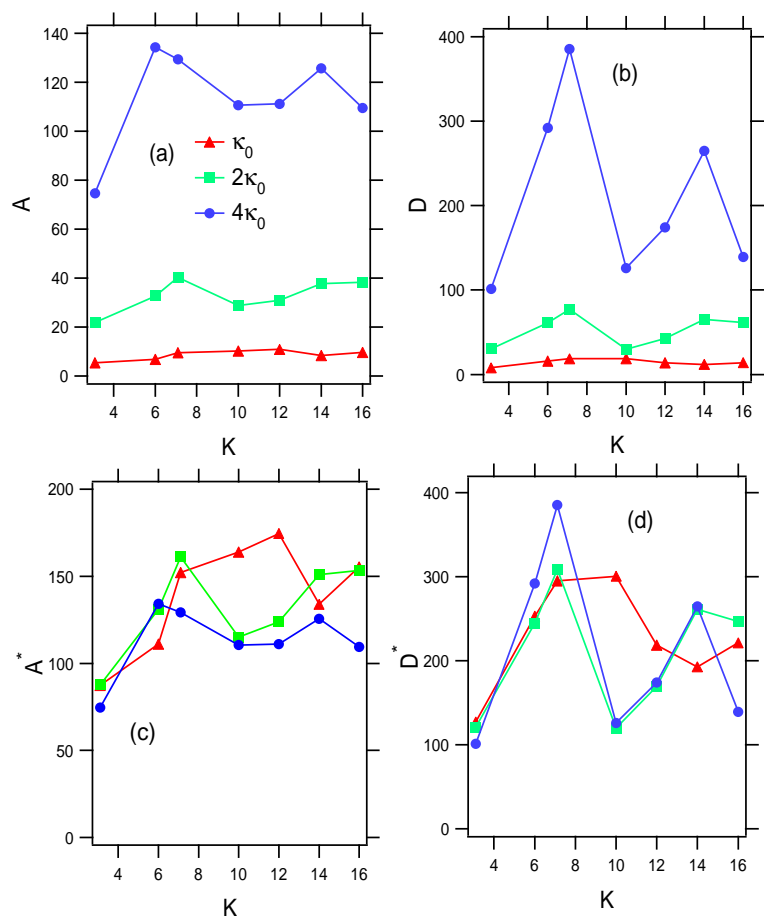

FIG. 3: (Color online) $K$-dependence of the coefficients (a) $A$ and (b) $D$ of SM with a fixed $\kappa(\equiv K / \hbar)$. The three cases for $\kappa=\kappa_{0}, 2 \kappa_{0}, 4 \kappa_{0}\left(\kappa_{0}=18\right)$ are shown from below. The scaled coefficients $A^{*}=A /\left(\kappa^{2} / \kappa_{\max }\right)$ and $D^{*}=D /\left(\kappa^{2} / \kappa_{\max }\right)$, where $\kappa_{\max }=4 \kappa_{0}$, are shown for $\kappa=\kappa 0,2 \kappa 0$ and, $4 \kappa 0$ in (c) and (d) of the lower panels, respectively. The three curves almost overlap.

sented by,

$$
p_{\xi} \propto\left(\frac{K}{\hbar}\right)^{2} \exp \left[\operatorname{const.\epsilon }\left(\frac{\mathrm{K}}{\hbar}\right)^{2}\right]
$$

in the quantum regime. Note that in a limit of $\epsilon \rightarrow 0, p_{\xi}$ becomes the localization length of the unperturbed SM $p_{\xi} \propto\left(\frac{K}{\hbar}\right)^{2}$ first proposed by Casati et al $[\underline{3}$.

Finally, we plot the coefficients $A$ and $D$ in Fig 5 as a function of $K / \hbar$ fixing $\hbar$ at two significantly different values $\hbar=0.56$ and 3.1 and changing $K$. The coefficients $A$ and $D$ are not only proportional to $(K / \hbar)^{2}$, but the two curves of $A$ and of $D$ overlaps by extrapolation, which again establishes the results given by Eqs.(10) and (11).

The results obtained above agrees entirely with the experimental results of the Manai et al.. They explained their results by applying self-consistent mean field theory (SCT) to the monochromatically perturbed SM, and they obtained $A \propto\left(\frac{K}{\hbar}\right)^{2}$, but $D \propto \frac{K}{\hbar}$, which is inconsistent with Eq.(11). A modified version of SCT of the localization naturally leading to the result Eq.(12) will be presented in Sect $\mathrm{VB}$. The modification is essential for the theoretical prediction of the localization length of AM discussed in the next section.
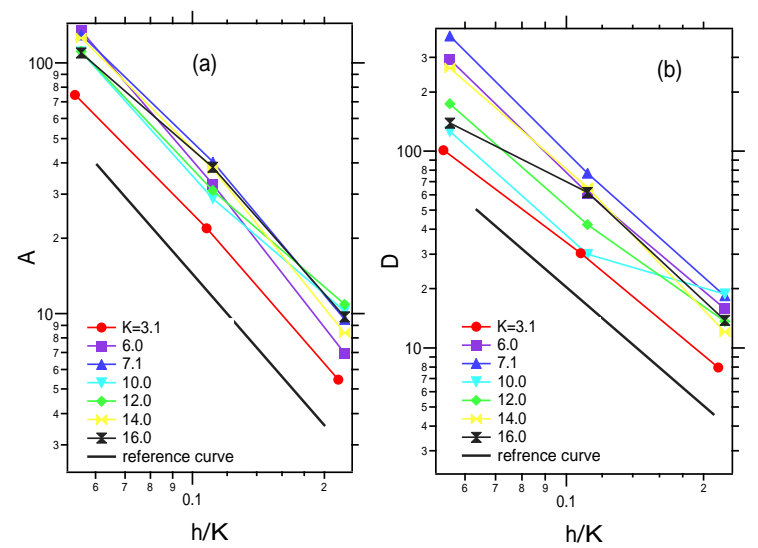

FIG. 4: (Color online) (a) $(\hbar / K)$-dependence of the coefficients (a) $A$ and (b) $D$ for some $K$ 's. Three cases of $\kappa=$ $\kappa_{0}, 2 \kappa_{0}, 4 \kappa_{0}\left(\kappa_{0}=18\right)$ are shown in the straight lines. Note that the axes are in logarithmic scale. The heavy line shows a straight line with slope of -2 corresponding to the $(\hbar / K)^{-2}$.

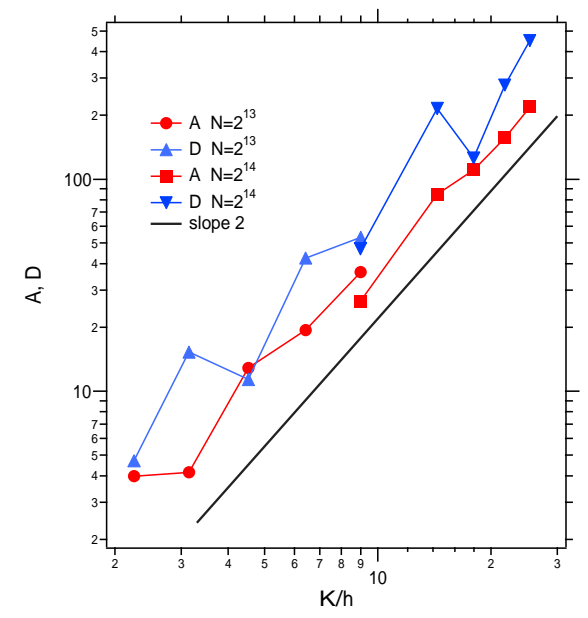

FIG. 5: (Color online) $(K / \hbar)$-dependences of the coefficients $A$ and $D$ when varying the combination of $K$ and $\hbar$ variously. Note that the axes are in logarithmic scale. The heavy line shows a straight line with slope of 2 corresponding to the $(\hbar / K)^{2}$.

\section{LOCALIZATION PHENOMENA IN MONOCHROMATICALLY PERTURBED AM}

In this section, we show the numerical results for the localization characteristics of the monochromatically perturbed AM. The scaling properties of LL is explored by varying disorder strength $W$ and perturbation strength $\epsilon$. 


\section{A. $W$-dependence}

It has been analytically found that in 1DDS the $W$-dependence of the LL of eigenstates behaves like $W^{-2}$ for weak disorder limit $W<<1$ by perturbation theory [24, 25] and it decreases obeying $1 / \log W$ in the strong disorder limit $W>>1$ [26, 27]. Therefore, if the $\mathrm{LL}$ is almost same as the dynamical localization length, we can expect the time-dependent spread of the initially localized wavepacket is suppressed around the LL, and the $W$-dependence of the saturated MSD behaves like $m_{2}(t)\left(=\xi_{0}^{2}\right) \sim W^{-4}$ in the weak disorder limit.

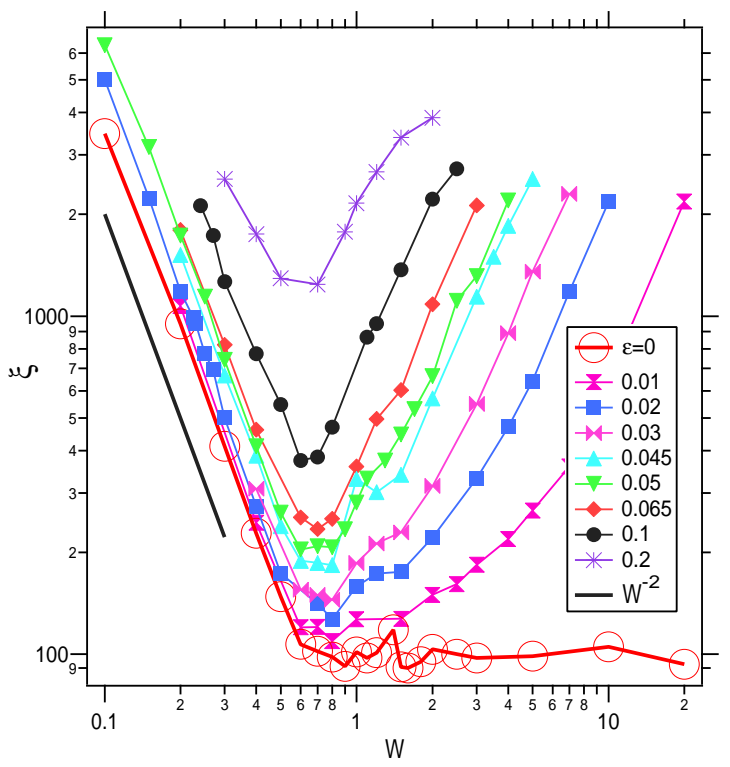

FIG. 6: (Color online) Localization length of the monochromatically perturbed AM as a function of disorder strength $W$ for various perturbation strength $\epsilon$. The unperturbed case $(\epsilon=0)$ is denoted by a thick line with large circles. Note that the axes are in the logarithmic scale.

Based on these facts, we investigate the localization properties of the wavepacket in the monochromatically perturbed AM. Figure [6] shows the $W$-dependence of the LL in the system for various perturbation strength $\epsilon$. First, let us focus on the unperturbed case $(\epsilon=0)$ for which a typical situation of localization in AM is expected to occur. It follows that the LL $\xi_{0}$ of the unperturbed case decreases like $W^{-2}$ in the weak disorder regime as expected, but the decrease ceases around a certain value denoted by $W^{*}$, that is,

$$
\xi_{0} \simeq \begin{cases}c_{0} W^{-2} & \left(W<W^{*}\right) \\ \xi_{0}^{*} & \left(W>W^{*}\right)\end{cases}
$$

where $c_{0}$ is a constant and $\xi_{0}^{*}=\xi_{0}\left(W^{*}\right)$. The result is consistent with the perturbation theory only for the limit $W<<1$, as mentioned in Sect II. Its reason is reconsidered with the Maryland transform in the next section, but very intuitively we can explain the presence of the characteristic value $W^{*}$ above which the $\xi_{0} \sim W^{-2}$ behaviour changes to $\xi_{0} \sim$ const by the periodic nature of the dynamical perturbation Eq. (2) in Sect III. Eq.(1) together with Eq.(2) allows us to interpret the original Hamiltonian of AM (set $\epsilon=0$ for simplicity) as the Anderson model Hamiltonian $T(\hat{p})+V(\hat{q}) / \tau$ to which the dynamical perturbation $(2 / \tau) \sum_{m=1}^{\infty} \cos (2 \pi m t / \tau)$ of the period $\tau$ is added. The latter induces a transition between the localized eigenstates of the Anderson model if the typical energy width $W$ of the localized states exceeds the minimal quantum unit $\hbar 2 \pi / \tau$ of the periodic perturbation. Accordingly, it is expected that the $\delta$-function weakens the localization effect when $W$ exceeds the characteristic value

$$
W^{*} \simeq \frac{2 \pi}{\tau} \hbar
$$

Taking $\hbar=1 / 8, \tau=1$, the above formula yields $W^{*} \sim$ 0.8 which is consistent with the characteristic value of Fig 6 above which the monotonous decrease obeying the $W^{-2}$-law ceases. Indeed, we have confirmed the change of the value $W^{*}$ obeys Eq.(14) by varying the period $\tau$.

The curves of the LL for various values of the perturbation strength $\epsilon$ is over-plotted in Fig 6. For $W<W^{*}$, the $W^{-2}$-dependence is stably maintained even for $\epsilon \neq 0$ but the LL increases with increase in the perturbation strength $\epsilon$ at least in the weak perturbation limit $\epsilon<<1$. On the other hand, for $W>W^{*}$, the LL grows up as the disorder strength $W$ increases if $\epsilon \neq 0$. In the next subsection, we look into the details of the $\epsilon$-dependence of the LL for the two regions, i.e., $W<W^{*}$ and $W>W^{*}$, to clarify their characteristics.

\section{B. $\epsilon$-dependence}

Figure 7(a) and (c) show the result of the $\epsilon$-dependence in the the monochromatically perturbed AMfor $W<W^{*}$ and $W>W^{*}$, respectively. It is obvious that the LL grows exponentially as the perturbation strength $\epsilon$ increases in the both cases. Therefore, in the same way as the case of SM the LL can be expressed as

$$
\xi \simeq D \exp \{A \epsilon\}
$$

The coefficient $D$ should be the LL at $\epsilon=0$ and so $D=$ $\xi_{0}$, whose characteristics are given as Eq.(13). The most interesting point is the $W$-dependence of the coefficient $A$ in the two characteristic regions, $W<W^{*}$ and $W>$ $W^{*}$. For $W<W^{*}$ the coefficient $A$ is almost independent of $W$, and $\xi W^{2} \propto \xi / \xi_{0}=\xi / D$ as a function of $\epsilon$ overlaps each other as shown in Fig $7(b)$. As a result, we can obtain the relation $\xi \sim c_{0} W^{-2} \exp \left\{c_{1} \epsilon\right\}$, where $c_{0}$ and $c_{1}$ are certain constants.

On the other hand, in the region $W>W^{*}$ peculiar to $\mathrm{AM}$, there is a trend that the coefficient $A$ increases with $W$ as seen in Fig](c). The inset in panel (c) shows the slope (coefficient $A$ ) of the data in the panel (c) determined by fitting in the range of $0.01<\epsilon<0.08$ using 

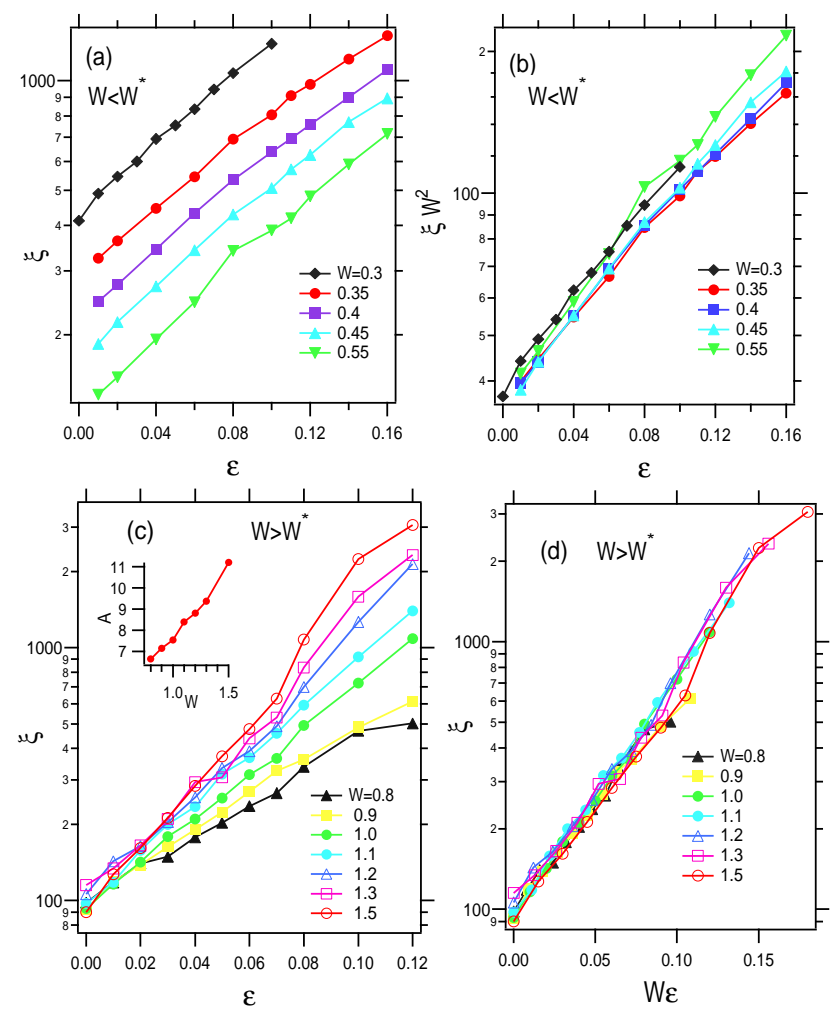

FIG. 7: (Color online) Localization length of the monochromatically perturbed AM as a function of perturbation strength $\epsilon$ for (a) $W<W^{*}$, (c) $W>W^{*}$, where $W^{*}=0.8$. (b)Plot of $\xi W^{2}$ as a function of $\epsilon$ for $W<W^{*}$. (d)Plot of $\xi$ as a function of $\epsilon W$ for $W>W^{*}$. Note that the all the vertical axes are in the logarithmic scale. The inset in panel (c) shows the plot of the coefficient $A$ as a function of $W$, which are estimated by linear fitting for data in the panel.

the method of least squares. The $W$-dependence of the slope increases almost linearly. Indeed, all the plots of the LL as a function of $W \epsilon$ overlap as shown in Fig:7(d) for $W \epsilon<<1$. The same scaling behaviours have been observed for the cases with different frequency $\omega_{1}$ as given in appendix $\mathrm{A}$

As a result, the LL $\xi$ of the monochromatically perturbed AM can be summarized as follows;

$$
\xi \simeq \begin{cases}c_{0} W^{-2} \exp \left\{c_{1} \epsilon\right\} & \left(W<W^{*}\right) \\ \xi_{0}^{*} \exp \left\{c_{2} W \epsilon\right\} & \left(W>W^{*}\right)\end{cases}
$$

where $c_{0}, c_{1}, c_{2}$ are numerical constants and $\xi_{0}^{*}$ is the saturated LL of the unperturbed case.

The $W$-dependence of the coefficient $A$ is very different from those in SM although the exponential growth with respect to $\epsilon$ is common.

Beyond $W^{*}$ the transition between the localized states due to the dynamical perturbation play the role of stopping the decrease of LL as is exhibited by Eq. (13). Recalling that the dynamical part of perturbation potential in $\mathrm{AM}$ is given by $\epsilon W v(n) \cos \left(\omega_{1} t\right)$, it is interesting to see the fact that the perturbation amplitude $W \epsilon$ does not influence the LL until $W$ exceeds $W^{*}$, which means that the effect of dynamical perturbation fully works only after the transition channel opens. Interpretation of Eq. (16) by SCT of the localization will be presented in next section.

\section{THEORETICAL EXPLANATION}

In this section, we first confirm the relationship among SM, AM and 2DDS by the Maryland transform [14]. Next, we give a theoretical explanation for the scaling properties obtained numerically in the last two sections is given based on the self-consistent mean-field theory (SCT) of the Anderson localization in 2DDS 22].

\section{A. autonomous representation and Maryland transformation}

We return to the two degrees of freedom unitaryevolution operator (5) which takes the monochromatic dynamical perturbation into account by the $J$-oscillator in an autonomous way.

$$
\hat{U}_{t o t}=\mathrm{e}^{-i \hat{A}} \mathrm{e}^{-i \hat{B}} \mathrm{e}^{-i \hat{C}},
$$

where

$$
\begin{aligned}
\mathrm{e}^{-i \hat{A}} & =\mathrm{e}^{-\frac{i}{\hbar}\left[T(\hat{p})+\omega_{1} \hat{J}\right] \tau}, \\
\mathrm{e}^{-i \hat{B}} & =\mathrm{e}^{-\frac{i}{\hbar} \epsilon \hat{V}(q) \cos \phi \tau}, \\
\mathrm{e}^{-i \hat{C}} & =\mathrm{e}^{-\frac{i}{\hbar} V(\hat{q}) \tau} .
\end{aligned}
$$

$\tau=1$ in this paper. We consider an eigenvalue equation,

$$
\hat{U}_{t o t}\left|u_{1}\right\rangle=\mathrm{e}^{-i \gamma}\left|u_{1}\right\rangle
$$

where $\gamma$ and $\left|u_{1}\right\rangle$ are the quasi-eigenvalue and quasieigenstate, respectively. This eigenvalue problem can be mapped into the tight-binding form by the Maryland transform, which provides with the foundation for applying the analysis developed for the 2DDS to our systems. This formulation further gives rise to some remarks about our approach.

For the SM, the eigenvalue equation we take the representation using eigenstate $|m\rangle(m \in \mathbb{Z})$ of momentum $\hat{p}$ and the action eigenstate $|j\rangle(j \in \mathbb{Z})$ of the $J$-oscillator as $u_{1}(m, j)=\left(\left\langle m|\otimes\langle j|) \mid u_{1}\right\rangle\right.$. Then by applying the Maryland transform, Eq.(21) is transformed into the eigenvalue equation of the following two-dimensional lattice system (tight-binding model) with aperiodic and singular on-site potential for newly defined eigenfunction $|u\rangle$ related to the original one $\left|u_{1}\right\rangle$ with an appropriate transform shown in an appendix B 


$$
\tan \left[\frac{\hbar^{2} m^{2} / 2+j \omega_{1} \hbar}{2 \hbar} \tau-\frac{\gamma}{2}\right] u(m, j)+\sum_{m^{\prime}, j^{\prime}}\left\langle m, j|\hat{t}| m^{\prime}, j^{\prime}\right\rangle u\left(m^{\prime}, j^{\prime}\right)=0
$$

where the transfer matrix element is

$$
\left\langle m, j|\hat{t}| m^{\prime}, j^{\prime}\right\rangle=\frac{1}{(2 \pi)^{2}} \int_{0}^{2 \pi} \int_{0}^{2 \pi} d q d \phi \mathrm{e}^{-i\left(m-m^{\prime}\right) q} \mathrm{e}^{i\left(j-j^{\prime}\right) \phi} \tan \left[\frac{K \cos q(1+\epsilon \cos \phi)}{2 \hbar} \tau\right] .
$$

This is the Maryland transformed eigenvalue equation including the additional degree of freedom contributing as the monochromatic perturbation in the monochromatically perturbed SM. The details of the derivation is given in appendix B. Of particular note is that in the semiclassical limit $\hbar \rightarrow 0$ the potential term become singular. Indeed, under the condition $|K \tau / \hbar|>\pi$ the transfer matrix element become the Fourier coefficient of a function having poles on the real axis and the transfer matrix ele- ment do not decay as $\left|m-m^{\prime}\right| \rightarrow \infty$ and so the analogy with the normal 2DDS is lost.

On the other hand, for the AM, we use the representation $u(n, j)(=(\langle n|\otimes\langle j|) \mid \bar{u}\rangle)$ based on the eigenstates $\mid q=n>$ of the site operator $\hat{n}$ and the eigenstates $|j\rangle$ of the operator $\hat{J}$, and the Maryland transformed eigenvalue equation becomes

$$
\tan \left[\frac{W v_{n}+j \omega_{1} \hbar}{2 \hbar} \tau-\frac{\gamma}{2}\right] u(n, j)+\sum_{n^{\prime}, j^{\prime}}\left\langle n, j|\hat{t}| n^{\prime}, j^{\prime}\right\rangle u\left(n^{\prime}, j^{\prime}\right)=0
$$

where

$$
\left\langle n, j|\hat{t}| n^{\prime}, j^{\prime}\right\rangle=\left\langle n, j\left|i \frac{e^{-i \epsilon W v(\hat{q}) \cos \phi \tau / \hbar}-e^{i 2 \cos (\hat{p} / \hbar) \tau / \hbar}}{e^{-i \epsilon W v(\hat{q}) \cos \phi \tau / \hbar}+e^{i 2 \cos (\hat{p} / \hbar) \tau / \hbar}}\right| n^{\prime}, j^{\prime}\right\rangle .
$$

In the case of $\epsilon \neq 0$, the evaluation of matrix elements is not easy since the stochastic quantity $v_{n}$ is contained in addition to both operators $\hat{q}$ and $\hat{p}$.

If we take $\epsilon=0$ as the simplest case of the hopping term in the transformed equation of $\mathrm{AM}$, it becomes

$$
\begin{aligned}
& \left\langle n, j|\hat{t}| n^{\prime}, j^{\prime}\right\rangle \\
= & \frac{1}{2 \pi} \int_{0}^{2 \pi} d p \mathrm{e}^{i\left(n-n^{\prime}\right) p} \tan \left[\frac{\cos (p / \hbar)}{\hbar} \tau\right],
\end{aligned}
$$

where $p=2 \pi \hbar k / N$. In the small $\tau$ limit the above equation results in an eigenvalue equation of the Anderson model with the nearest neighbouring hopping because $\tan (x) \simeq x$. On the other hand as $W$ increases such that $\tau W / 2 \hbar$ exceeds $\pi / 2$, the range of the on site potential of Eq.(24) covers the maximal range beyond which the distribution of the onsite potential do not change. This is an alternative explanation for the saturation of the localization length beyond $W^{*}$, which has been discussed in subsection IVA

Furthermore, from the Maryland transformed Eqs. (22) and (24), with $\epsilon=0$ the relationship between AM and SM can also be roughly estimated. In case of nearest neighbouring hopping for AM, the disorder strength increases with $W / \hbar$. On the other hand, the hopping strength increases with increase of $K / \hbar$ for $K / \hbar<<1$ in the case of SM. If the hopping strength is normalized to be unity, the disorder strength becomes proportional to $\hbar / K$. Accordingly, we can also see that the correspondence is roughly given as,

$$
\frac{W}{\hbar} \Leftrightarrow \frac{\hbar}{K} .
$$

\section{B. Interpretation of the scaling properties based on self-consistent theory of the localization}

Using the self-consistent theory of the mean-field approximation for the localization in the anisotropic 2DDS, we interpret the scaling characteristics on the numerical results for SM obtained in Sect $\amalg$ and AM in Sect IV. respectively.

Let $D_{\mu}(\omega)$ be the dynamical diffusion constant in the $\mu$ direction $(\mu=1,2)$. It is modified from the bare diffusion constant $D_{\mu}^{(0)}$ due to the destructive quantum interference induced by the backward scattering process of potential and is determined by the following self-consistent 
equation:

$$
\frac{D_{\mu}(\omega)}{D_{\mu}^{(0)}}=1-\frac{1}{\pi \rho} \frac{D_{\mu}(\omega)}{D_{\mu}^{(0)}} \sum_{q_{1}, q_{2}} \frac{1}{-i \omega+\sum_{\nu=1}^{2} D_{\nu}(\omega) q_{\nu}^{2}}
$$

The second term in the righthand side indicates the reduction by the quantum interference effect. ( $\rho$ is the density of states.) In the localized phase, the $\omega$-dependent diffusion coefficient has a form $D_{\mu}(\omega) \propto-i \omega$, and is related to a scale of the length $\xi(\omega)$ in the infinite system as follows:

$$
\xi_{\mu}(\omega)^{2}=D_{\mu}(\omega) /(-i \omega),
$$

which indeed becomes the localization length $\xi(\omega=0)($ or $\left.p_{\xi}(\omega=0)\right)$, in the limit of $\omega \rightarrow 0$. Here the summation over the wavenumber $q_{\mu}$ is done up to the upper cutoff decided by the inverse of the characteristics length $\ell_{\mu}$ 's which are important parameters discussed below in detail. Then Eq.(29) in the $\mu$-direction is rewritten by an integral form

$$
\begin{aligned}
& \frac{\xi_{\mu}(\omega)^{2}}{\ell_{\mu}^{2}}\left(-i \omega t_{\mu}\right) \\
= & 1-\frac{\xi_{\mu}(\omega)^{2} t_{\mu}}{\ell_{\mu}^{2}} \frac{1}{\xi_{1}(\omega) \xi_{2}(\omega)} \Xi\left[\frac{\xi_{1}(\omega)}{\ell_{1}}, \frac{\xi_{2}(\omega)}{\ell_{2}}\right],
\end{aligned}
$$

where $t_{\mu}=\ell_{\mu}^{2} / D_{\mu}^{(0)}$ means the localization time, and

$$
\begin{aligned}
& \Xi\left[\frac{\xi_{1}(\omega)}{\ell_{1}}, \frac{\xi_{2}(\omega)}{\ell_{2}}\right] \\
= & \tilde{c} \int_{0}^{\xi_{1}(\omega) / \ell_{1}} \int_{0}^{\xi_{2}(\omega) / \ell_{2}} d Q_{1} d Q_{2} \frac{1}{1+Q_{1}^{2}+Q_{2}^{2}},
\end{aligned}
$$

where $\tilde{c}$ is an appropriate numerical factor of $O(1)$. Here, the characteristic length $\ell_{\mu}$ of the integration range is usually taken as the mean free path, but in this paper we will propose different characteristic length as shown below.

Taking the case of SM as an example, we show the difference of the length proposed in this paper (maximum distance) from the ordinary length (minimum distance) as the characteristic length $\ell_{\mu}$. Let the kicked system with the characteristic length $\ell_{1}$ be the main system and the $J$-oscillator with the characteristic length $\ell_{2}$ the subsystem. The ordinary selection for $\ell_{\mu}$ is the minimum distance given as the hopping length $p(s+1)-p(s)=$ $K \sin q_{s}$ for a single step evolution from Eq.(77). The mean square values are

$$
\begin{aligned}
& \left(\ell_{1} \hbar\right)^{2}=K^{2}\left\langle\sin ^{2} q_{s}\right\rangle=K^{2} / 2, \\
& \left(\ell_{2} \hbar\right)^{2}=K^{2} \epsilon^{2}\left\langle\cos ^{2} q_{s}\right\rangle \sin ^{2} \phi_{s}=K^{2} \epsilon^{2} / 4 .
\end{aligned}
$$

Here $\langle\ldots\rangle$ indicates the quantum mechanical average with respect to the initial state. These correspond to the so called mean free path. An another candidate is the maximum distance reachable in an infinite time scale represented by the total hopping length $p(\infty)-p(0)=$ $\lim _{s \rightarrow \infty} K \sum_{s^{\prime}<s} \sin q_{s^{\prime}}$. Now we are considering the weak perturbation limit of $\epsilon$ in which the kicked system is decoupled from the subsystem, and maintains the diffusive motion as $m_{2}(s)=\left\langle(p(s)-p(0))^{2}\right\rangle=D_{c l} s$, where $D_{c l}$ is the classical diffusion constant, until the localization time which should coincides with the number of states in the maximum length ,i.e. $\ell_{1}$ of the main system. (This corresponds to the so-called Heisenberg time, and precisely a numerical factor must be multiplied but we ignore it.)

On the other hand, the $J$-oscillator (the color degrees of freedom) also exhibits a passive diffusive motion up to

$$
t_{2}=t_{1}=\ell_{1},
$$

being driven by the same force as the main system. (See Eq.(17).) From Eq.(7) the MSD's are expressed:

$$
\begin{aligned}
\lim _{s \rightarrow \infty}\left\langle(p(s)-p(0))^{2}\right\rangle & =\lim _{T \rightarrow \infty} \sum_{s \leq T} D_{1 s}, \\
\lim _{s \rightarrow \infty}\left\langle(J(s)-J(0))^{2}\right\rangle & =\lim _{T \rightarrow \infty} \sum_{s \leq T} D_{2 s},
\end{aligned}
$$

where the time-dependent diffusion constants $D_{1 s}$ and $D_{2 s}$ are defined by

$$
\begin{aligned}
D_{1 s} & =K^{2}\left[\left\langle\sin ^{2} q_{s}\right\rangle+\operatorname{Re} \sum_{s^{\prime}<s}\left\langle\sin q_{s^{\prime}} \sin q_{s}\right\rangle\right] \\
D_{2 s} & =K^{2} \epsilon^{2}\left[\left\langle\cos ^{2} q_{s}\right\rangle \sin ^{2} \phi_{s}\right. \\
& \left.+\operatorname{Re} \sum_{s^{\prime}<s}\left\langle\cos q_{s^{\prime}} \cos q_{s}\right\rangle \sin \phi_{s^{\prime}} \sin \phi_{s}\right] .
\end{aligned}
$$

As the maximum diffusion lengths, $\ell_{1}$ and $\ell_{2}$, are proposed

$$
\begin{aligned}
& \ell_{1}^{2} \hbar^{2}=\lim _{s \rightarrow \infty}\left\langle(p(s)-p(0))^{2}\right\rangle, \\
& \ell_{2}^{2} \hbar^{2}=\lim _{s \rightarrow \infty}\left\langle(J(s)-J(0))^{2}\right\rangle .
\end{aligned}
$$

The time-dependent diffusion constants are a given as $D_{1 s}=D_{1}^{(0)} \hbar^{2}=D_{c l}$ and $D_{2 s} \equiv D_{2}^{(0)} \hbar^{2} \sim D_{c l} \epsilon^{2} / 2$ until $t_{1}=t_{2}=\ell_{1}$, but both collapse to zero beyond it. Then Eqs.(36)-(40) lead to

$$
\begin{aligned}
& \ell_{1}^{2}=D_{1}^{(0)} \ell_{1}, \\
& \ell_{2}^{2}=D_{2}^{(0)} \ell_{1} .
\end{aligned}
$$

This is a general relation applicable to the case of AM as will be used later. If we suppose the Markovian limit that the autocorrelation function of the force term coming from the main system is given by $\left\langle\cos q_{s} \cos q_{s^{\prime}}\right\rangle=$ $\left\langle\sin q_{s} \sin q_{s^{\prime}}\right\rangle=\delta_{s, s^{\prime}} / 2$, then $D_{1}^{(0)}=D_{c l} / \hbar^{2}=K^{2} / 2 \hbar^{2}$, $D_{2}^{(0)}=\epsilon^{2} D_{1}^{(0)} / 2$, and $\ell_{1,2}$ is given as

$$
\begin{aligned}
& \ell_{1}=D_{1}^{(0)}=\frac{D_{c l}}{\hbar^{2}}, \\
& \ell_{2}=\frac{\epsilon}{\sqrt{2}} D_{1}^{(0)} .
\end{aligned}
$$


As the fundamental distance $\ell_{1}, \ell_{2}$, we use these "maximal distance" rather than the "minimal distance" taken by the Manai et al. [15]. We further remark that one can easily check the one-dimensional version of Eq.(31) can give the localization length of the standard map $\frac{D_{c l}}{\hbar^{2}}$ only by assuming $\ell_{1}=D_{1}^{(0)}=\frac{D_{c l}}{\hbar^{2}}$.

Under the above setting, Eq.(31) tells that the factor $\frac{\xi_{\mu}(\omega)^{2}}{\ell_{\mu}^{2}} t_{\mu}$ is independent of $\mu$, which means that

$$
\frac{\xi_{1}(\omega)}{\ell_{1}}=\frac{\xi_{2}(\omega)}{\ell_{2}}
$$

because $t_{\mu}=t_{1}$ from Eq.(35). Carrying out the integral in the r.h.s of Eq.(31) for $\mu=1$, using the above relation, one has

$$
\begin{aligned}
& \frac{\xi_{1}(\omega)^{2}}{\ell_{1}^{2}}\left(-i \omega \ell_{1}\right) \\
= & 1-\frac{\tilde{c}}{\ell_{2}} \log \left[1+\frac{\xi_{1}(\omega)^{2}}{\ell_{1}^{2}}\right] .
\end{aligned}
$$

Taking a limit $\omega \rightarrow 0$ and organizing the expressions, the localization length $\xi_{1}(0)$ becomes

$$
\xi_{1}(\omega=0) \sim \ell_{1} \mathrm{e}^{\ell_{2} / 2 \tilde{c}},
$$

where $\ell_{1}=\frac{D_{c l}}{\hbar^{2}}=\frac{K^{2}}{2 \hbar^{2}}$ and $\ell_{2}=\frac{\epsilon D_{c l}}{\sqrt{2} \hbar^{2}}=\epsilon \frac{K^{2}}{2^{3 / 2} \hbar^{2}}$ are two selected characteristic lengths, and $\tilde{c}$ is a suitable constant. This corresponds to the localization length in SM under the monochromatic perturbation in the previous sections. We remark that in the case of typical isotropic 2DDS the characteristic length is $\ell_{1}=\ell_{2}(=\ell)$ can be identified with the mean free path $\ell_{m f p}$ and Eq.(47) yields the well-known result $\xi_{2 d d s} \sim \ell_{m f p} \mathrm{e}^{\pi \ell_{m f p} / 2}$.

We can straightforwardly apply the above analysis to the perturbed AM. In a similar way as in the case of $\mathrm{SM}$, the diffusion length of the $J$-oscillator is obtained by replacing the term $K \epsilon \cos q_{s} \sin \phi_{s}$ in Eq. (77) coming from the interaction potential $K \epsilon \cos q_{s} \cos \phi_{s}$ by the term $W \epsilon v\left(q_{s}\right) \sin \phi_{s}$ in the interaction potential of the $\mathrm{AM}$ as,

$$
\ell_{2}^{2} \hbar^{2} \sim W^{2} \epsilon^{2} \sum_{s, s^{\prime}}\left\langle v\left(q_{s}\right) v\left(q_{s^{\prime}}\right)\right\rangle\left\langle\sin \phi_{s} \sin \phi_{s^{\prime}}\right\rangle .
$$

Since the diffusion time of the kicked system is given by $\ell_{1}$

$$
\ell_{2}^{2} \hbar^{2} \sim W^{2} \epsilon^{2} \ell_{1} / 2 .
$$

Accordingly, in the case of AM

$$
\ell_{1} \simeq \begin{cases}1 / W^{2} & \left(W<W^{*}\right) \\ 1 / W^{* 2} & \left(W>W^{*}\right)\end{cases}
$$

Therefore,

$$
\ell_{2} \sim \epsilon \sqrt{W^{2} \ell_{1} / 2} \simeq \begin{cases}\epsilon / \sqrt{2} & \left(W<W^{*}\right) \\ \epsilon W / \sqrt{2} & \left(W>W^{*}\right) .\end{cases}
$$

It follows that when these are used for the expression (47), results are consistent with that obtained by the numerical calculation in the previous sections.

In the SM, it also means that what the Manai et al. used to explain the experimental results could be derived directly from the theoretical considerations by our selection for the characteristic lengths as the cut-off of the integral of SCT of the localization. Our hypothesis Eq. (40) is more vital in SM dynamically perturbed by more than two colors, in which a localization-delocalization transition occurs [11]. Indeed, it predicts precise parameter dependence of the critical value of $\epsilon$ numerically observed [12].

\section{DIFFUSION CHARACTERISTICS}

Up to the previous section, we investigated localization characteristics in a relatively small perturbation regime in which the LL can be decided numerically. The exponential growing rate of the localization length is enhanced by decreasing $\hbar$ or by increasing $W$ for SM and $\mathrm{AM}$, respectively. It is quite interesting to see the transient behaviour on the way to the final localization in the large limit of the exponentially enhanced LL. In the case of $\mathrm{AM}$, the region $W>W^{*}$ is focused on, because it is an essentially new region peculiar to the quantum map in which the quantum hopping is assisted by the kick perturbation. On the other hand, in the case of SM the limit $\hbar \rightarrow 0$ is of interest because it is the semiclassical limit in which the LL is much enhanced, and classical chaotic diffusion may be observed at least in the transient process, as has been first examined in coupled SM [28].

Figure $8(\mathrm{a})$ and (b) show the time-dependence of the MSD obtained for SM and AM by increasing the perturbation strength $\epsilon$, where $\hbar$ is fixed at a sufficiently small value for SM, and $W$ is fixed to a large value such that $W \gg W^{*}$ for AM. It becomes localized if $\epsilon$ is small, but as $\epsilon$ is increased larger, a diffusive behaviour emerges over a long time scale, which is apparently different from the monotonically localizing behaviour typically seen in the Fig! in Sect!

To observe the diffusive behaviour qualitatively the time-dependent diffusion coefficient defined by

$$
D(t)=\frac{d \bar{m}_{2}(t)}{d t},
$$

is convenient, where $\bar{m}_{2}(t)$ indicates a smoothed curve over a sufficiently long section of time including $t$. Figures $8(\mathrm{c})$ and $(\mathrm{d})$ show the time-dependence of $D(t)$ for the SM and AM, respectively. The time-dependent diffusion coefficient decreases with time being accompanied with fluctuation, but for sufficiently large $\epsilon$ the decrease of $D(t)$ is so slow that its variation is detectable only in the logarithmic time scale. In the early stage it seems to decreases linearly in logarithmic time scale. We remark that in the case of SM, $D(t)$ agrees with the classical diffusion coefficient $D_{c l}$ in the very initial stage, reflecting 

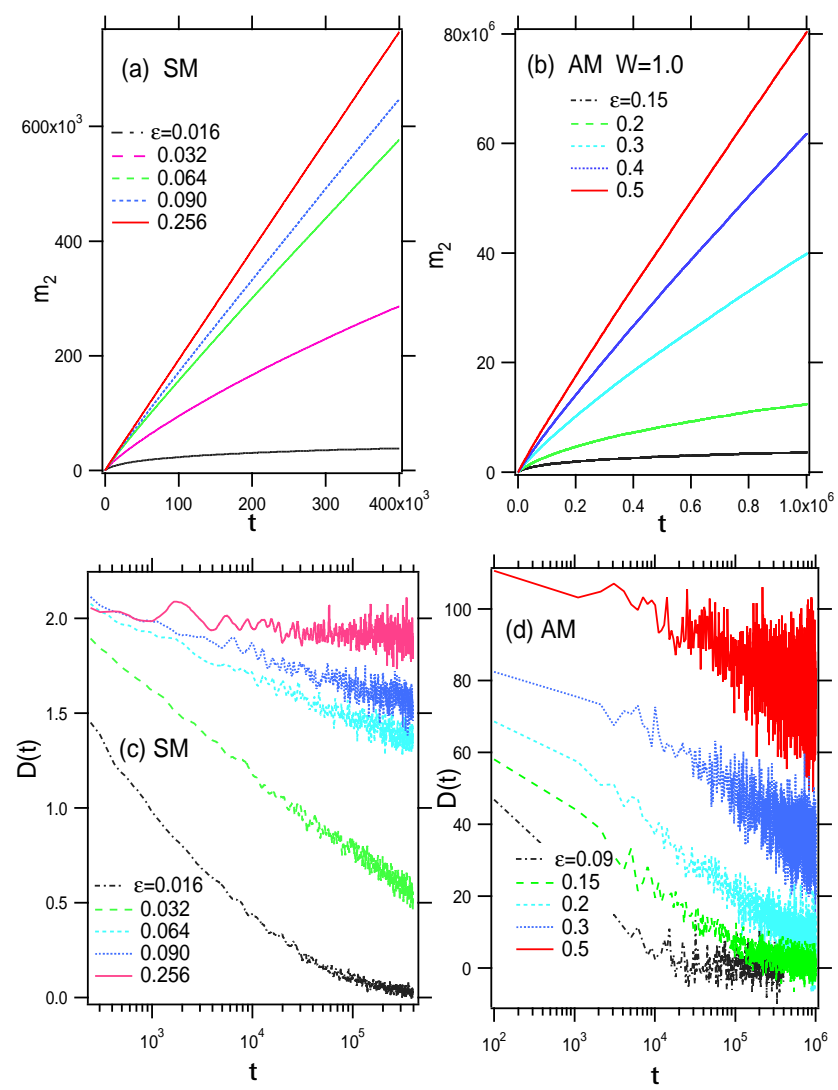

FIG. 8: (Color online) The time-dependence of the MSD and the diffusion coefficient $D(t)$ for relatively large perturbation strength. (a)Monochromatically perturbed SM for $K=3.1$ with some $\epsilon \mathrm{s}$. (b)Monochromatically perturbed AM for $W=$ 1.0 with some $\epsilon$ s. (c)(d) The $D(t)$ of the SM and the AM, respectively. Note that the horizontal axes of the $D(t)$ are in logarithmic scale.

the quantum-classical correspondence within the Ehrenfest time.

Next we characterize the $\epsilon$-dependence of the timedependent diffusion coefficient $D(t)$. Consider the time evolution up to $t=T$. As is shown in Fig $8 D(t)$ takes the minimum value at $t=T$, and $D(T)=0$ means that the wavepacket have localized until $t=T$. We are concerned with $D(t)$ after a very long time evolution, but we can not now specify the scale of $T$ on which the dynamics of localization process is characterized. At present, we tentatively take the time scale $T$ as long as our numerical run time allows, and we represent the minimum value $D(T) . \quad T$ is fixed at $5 \times 10^{5}-10^{6}$ steps. The results are plotted as the function of the perturbation strength $\epsilon$ for three values of $W(\mathrm{AM})$ and $\hbar(\mathrm{SM})$ in Figs 9 (a) and 10(a), respectively. For the SM we also plot $D(0)$, which is the maximum value of $D(t)$ and mimics the classical diffusion constant $D_{c l}$, in order to show explicitly the range scanned by $D(t)$ in the time interval $0 \leq t \leq T$. We plot also the classical diffusion coefficient $D_{c l}$ as a function of $\epsilon$. It follows that both in SM and AM the $D(T)$ gradually rise with $\epsilon$, and later it increases rapidly.
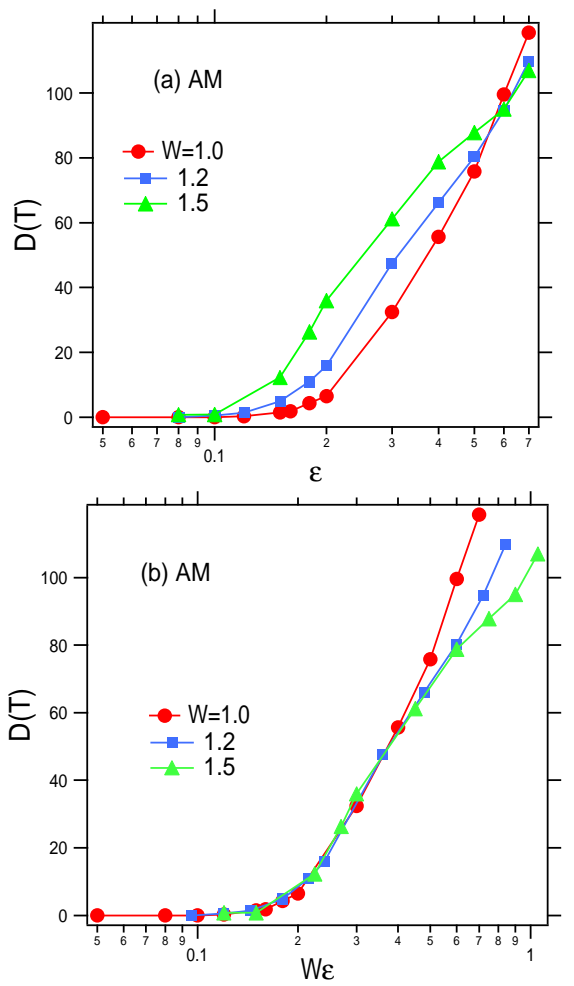

FIG. 9: (Color online) (a)The time-dependent diffusion coefficient $D(T)$ at $T=1 \times 10^{6}$ as a function of $\epsilon$ in the monochromatically perturbed AM with $W=1.0,1.2,1.5$. (b) The $D(T)$ as a function of $W \epsilon$. Note that the horizontal axes are in logarithmic scale.

In the AM, the $D(T)$ curve as a function of $\epsilon$ shifts upward with $W$, which is consistent with the dependence of LL on $\epsilon$ and $W$ as discussed in the SecIV In fact, taking the parameter $W \epsilon$ instead of $\epsilon$ the curves of diffusion coefficient $D(T)$ in Fig 9) a are all well-overlapped, as shown in Fig 9(b) if $\epsilon W$ is not too large. Recalling the result of the previous Sect IV that the single parameter $W \epsilon$ controls the LL, it is quite natural that the diffusion coefficients are also decided only by the combined parameter $W \epsilon$.

Finally we discuss the very important feature of SM which is not seen in AM. It is the existence of the semiclassical regime which emerges for small $\hbar$, as was shown in coupled SM.[28]. As mentioned above, $D(0)$ mimics the classical diffusion coefficient, however, $D(t)$ decreases as is shown in Fig 8 (c), and the decaying rate in $\log t$ scale decreases with $\epsilon$, and there exists a characteristic value $\epsilon_{c}$ beyond which the decay becomes extremely small and so $D(T) \sim D(0) \sim D_{c l}$. Indeed, Fig 10(a) exhibits that with increase in $\epsilon, D(T)$ increases rapidly, and beyond a certain $\epsilon=\epsilon_{c}$ it forms a plateau on which $D(T)$ keeps almost constant level. On the plateau the difference $D(T)-D(0)\left(\sim D_{c l}\right)$ is small, and $D(T)$ approaches 

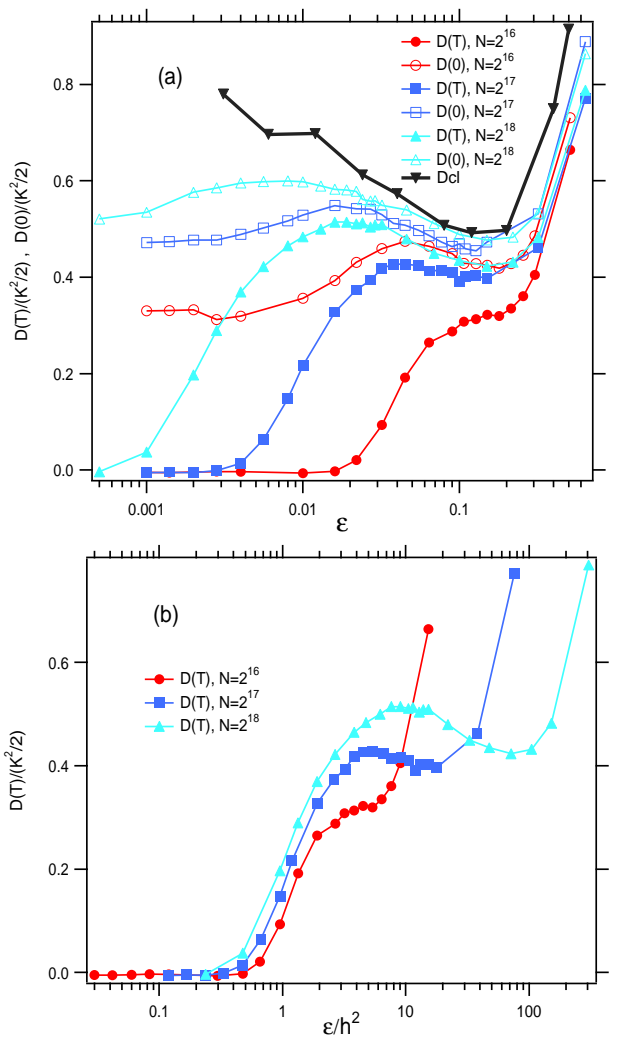

FIG. 10: (Color online) (a)The time-dependent diffusion coefficient $D(T) /\left(K^{2} / 2\right)$ at $T=4 \times 10^{5}$ as a function of $\epsilon$ in the monochromatically perturbed SM with $\hbar=\frac{2 \pi 1234}{2^{16}}$, $\hbar=\frac{2 \pi 1234}{2^{17}}, \hbar=\frac{2 \pi 1234}{2^{18}}$. Here $K=3.1$ for all data. (b) The $D(T)$ as a function of $\epsilon / \hbar^{2}$. Note that the horizontal axes are in logarithmic scale.

closer to $D_{c l}$ as $\hbar \rightarrow 0$. Thus we call the plateau as the "classical plateau" of the time-dependent diffusion coefficient. With further increase of $\epsilon$, the classical diffusion rate is enhanced and $D(T)$ takes off from the plateau following the enhanced $D_{c l}$ closely. Evidently, the classical plateau and the threshold $\epsilon_{c}$ shift toward smaller side of $\epsilon$ as $\hbar$ decreases. In the plots of $D(T)$ as the function of the scaled parameter $\epsilon / \hbar^{2}$ shown in Fig 10(b), the left edges of the plateaus for different $\hbar$ s coincide, which means that $\epsilon_{c} \propto \hbar^{2}$. Figure 10(b) also implies that $D(T)$ is controlled by $\epsilon / \hbar^{2}$, as is the case of the localization length of Eq. (12). Thus the quantum regime we introduced without definition previously should be

$$
\epsilon<\epsilon_{c}\left(=C \times \hbar^{2}\right)
$$

where the constant $C$ depends on $K$ such as $C \propto K^{2}$, but we have not confirmed it yet. The localization characteristics of the SM discussed in the previous sections have been confirmed only in the quantum regime. It is still open to question whether or not the localization characteristics represented by Eq. (12) is valid in the semiclassical regime.
The dynamical problems related to the localization process such as the existence of characteristic time leading to the localization and/or the existence of dynamical scaling property are still an interesting unclarified issue, particularly when the LL is extremely large for $\epsilon W \gg 1$ in AM with $W>W^{*}$ and for $\hbar \rightarrow 0$ in SM, respectively.

\section{CONCLUDING REMARKS}

We investigated the dynamical localization of the SM (standard map) and the AM (Anderson map) which are dynamically perturbed by a monochromatically periodic oscillation, and the parameter dependence of the dynamical localization length has been clarified by extensive numerical simulation and theoretical considerations. Under suitable conditions such systems could be identified with a 2DDS (two-dimensional disordered system) by using the so-called Maryland transform.

The dynamical localization length (LL) was determined by the MSD computed by the numerical wavepacket propagation. We emphasize that the SM is treated in the quantum regime, where the perturbation strength $\epsilon$ is smaller than a characteristic value proportional to $\hbar^{2}$. The LL increases exponentially with respect to $\epsilon$ in both perturbed SM and AM. It was further scaled by using the dynamical localization length of the unperturbed system in the case of SM, which is consistent with experimental results. On the other hand, in the case of AM, it was scaled by the disorder strength $W$. There exists the threshold of the disorder strength $W^{*}$ at which a marked change of $W$-dependence of the dynamical localization length occurs. In the region, $W<W^{*}$, the ordinary localization in 1DDS occurs, whereas new region, $W>W^{*}$, peculiar to the quantum map emerged where the localization length increases with the disorder strength $W$ due to the kicked perturbation.

Next, we showed that all the numerically observed scaling characteristics mentioned above can be reproduced in a unified manner by the self-consistent mean-field approximation theory developed for the localization of the 2DDS by introducing a new fundamental characteristic lengths as the cut-off length. This fact strongly suggests that the monochromatically perturbed SM and AM has essentially the same physical origin for the exponentially enhanced localization length.

Finally the transient diffusive behaviour toward the dynamical localization was investigated in the large limit of localization length. In both cases of SM and AM, the transient diffusion coefficient also follows the same scaling rule as the localization rule, but in the case of SM the "classical plateau" exists in the semiclassical regime, in which the compatibility of the quantum localization with the classical chaotic diffusion is quite interesting. Indeed, different type of localization which can not be captured by the above mentioned "unified picture" may emerges in the semiclassical regime. These are interesting problems still open to question. 
The Anderson map asymptotically approach to the original Anderson model in the limit of $\tau \rightarrow 0$ as mentioned in Sect II. Whether or not the result in this paper is true even in the time-continuous version (Anderson model) is also an interesting future problem.

\section{Appendix A: other numerical data the perturbed AM}

Figure 11 displays the localization length as a function of scaled perturbation strength $\epsilon W$ in the monochromatically perturbed $\mathrm{AM}$ with the frequencies $\omega_{1}^{(2)}=\sqrt{2}-1$, $\omega_{1}^{(3)}=1+1 / \sqrt{17}$ different from one in the text. At least the scaling of the localization length is a stable result even for the frequencies. It follows that for $W>W^{*}$ the scaled $\epsilon$-dependence is overlapping with each others. Also, although not shown here, for $W<W^{*}$ the $W$-dependence of the localization length in the cases also behaves similarly to that in the text.

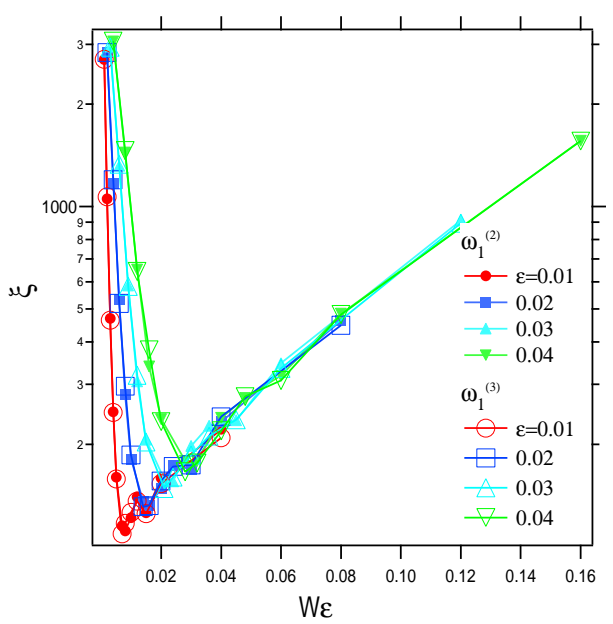

FIG. 11: (Color online) The localization length as a function of scaled perturbation strength $\epsilon W$ in the monochromatically perturbed AM for $\epsilon=0.01,0.02,0.03,0.04$ with perturbation frequencies $\omega_{1}^{(2)}=\sqrt{2}-1, \omega_{1}^{(3)}=1+1 / \sqrt{17}$. All are displayed together.

\section{Appendix B: autonomous representation and Maryland transform}

The eigenvalue problem Eq.(21) can be mapped into the tight-binding form by Maryland transform through the following states $\left|u_{2}\right\rangle,\left|u_{1}\right\rangle$ and Hermite matrices $\hat{t}, \hat{w}$;

$$
\begin{aligned}
& \left|u_{2}\right\rangle=\mathrm{e}^{-i \hat{B}} \mathrm{e}^{-i \hat{C}}\left|u_{1}\right\rangle \\
& \left|u_{1}\right\rangle=\mathrm{e}^{-i(\hat{A}-\gamma)}\left|u_{2}\right\rangle, \\
& \frac{1-i \hat{t}}{1+i \hat{t}}=\mathrm{e}^{-i \hat{B}} \mathrm{e}^{-i \hat{C}}, \\
& \frac{1-i \hat{w}}{1+i \hat{w}}=\mathrm{e}^{-i(\hat{A}-\gamma)} .
\end{aligned}
$$

That is,

$$
\begin{aligned}
\hat{t}(\hat{q}, \hat{p}, \phi) & =-i \frac{1-\mathrm{e}^{-i \hat{B}} \mathrm{e}^{-i \hat{C}}}{1+\mathrm{e}^{-i \hat{B}} \mathrm{e}^{-i \hat{C}}} \\
\hat{w}(\hat{p}, \hat{J}) & =-i \frac{1-\mathrm{e}^{-i(\hat{A}-\gamma)}}{1+\mathrm{e}^{-i(\hat{A}-\gamma)}}=\tan \left[\frac{(\hat{A}-\gamma)}{2}\right]
\end{aligned}
$$

Then the tight-binding form of the eigenvalue problem becomes

$$
\left(\tan \left[\frac{(\hat{A}-\gamma)}{2}\right]+\hat{t}(\hat{q}, \phi)\right)|\bar{u}\rangle=0
$$

where

$$
\begin{aligned}
(1+i \hat{t})^{-1}\left|u_{1}\right\rangle & =(1-i \hat{t})^{-1}\left|u_{2}\right\rangle \\
& =(1-i \hat{t})^{-1} \mathrm{e}^{i(\hat{A}-\gamma)}\left|u_{1}\right\rangle \\
& \equiv|\bar{u}\rangle
\end{aligned}
$$

and

$$
|\bar{u}\rangle=\left(\left|u_{1}\right\rangle+\left|u_{2}\right\rangle\right) / 2 .
$$

$\hat{t}=\tan [\hat{C} / 2]$ when $\epsilon=0$. We can select a convenient representation for the eigenvalue equation (B7). In this case, we dealt with the monochromatic perturbation in the autonomous representation, but the extension to the case of multicolor perturbation can be easily done.

For the SM, the eigenvalue equation in the representation by $u(m, j)=\langle m, j \mid \bar{u}\rangle$ based on the eigenstates $|m, j\rangle=|m\rangle \otimes|j\rangle$ of $\hat{p}$ and $\hat{J}$, respectively, is given by the following two-dimensional lattice system with aperiodic and singular on-site potential;

$$
\tan \left[\frac{\hbar^{2} m^{2} / 2+j \omega_{1} \hbar}{2 \hbar} \tau-\frac{\gamma}{2}\right] u(m, j)+\sum_{m^{\prime}, j^{\prime}}\left\langle m, j|\hat{t}| m^{\prime}, j^{\prime}\right\rangle u\left(m^{\prime}, j^{\prime}\right)=0
$$


where the transfer matrix element is given by Eq. (23) in the main text.

On the other hand, for the monochromatically perturbed AM, using

$$
\begin{aligned}
\hat{A} & =\left(W v(\hat{q})+\omega_{1} \hat{J}\right) \tau / \hbar, \\
\hat{B} & =\epsilon W v(\hat{q})(\cos \phi) \tau / \hbar \\
\hat{C} & =2 \cos (\hat{p} / \hbar) / \hbar
\end{aligned}
$$

$$
\left(\tan \left[\frac{\left(\hat{W}(\hat{q})+\omega_{1} \hat{J}-\gamma\right) \tau}{2}\right]+\hat{t}(\hat{p}, \hat{q}, \phi)\right)|\bar{u}\rangle=0
$$

If we use the representation $u(n, j)(=(\langle n|\otimes\langle j|) \mid \bar{u}\rangle)$ based on the eigenstates $|q=n\rangle$ of the site $\hat{n}$ and $|j\rangle$ of the $\hat{J}$, it becomes

$$
\tan \left[\frac{W v_{n}+j \omega_{1} \hbar}{2 \hbar} \tau-\frac{\gamma}{2}\right] u(n, j)+\sum_{n^{\prime}, j^{\prime}}\left\langle n, j|\hat{t}| n^{\prime}, j^{\prime}\right\rangle u\left(n^{\prime}, j^{\prime}\right)=0
$$

where

$$
\begin{aligned}
\left\langle n, j|\hat{t}| n^{\prime}, j^{\prime}\right\rangle & =\left\langle n, j\left|i \frac{e^{-i \hat{B}}-e^{i \hat{C}}}{e^{-i \hat{B}}+e^{i \hat{C}}}\right| n^{\prime}, j^{\prime}\right\rangle \\
& =\left\langle n, j\left|i \frac{e^{-i \epsilon W v(\hat{q}) \cos \phi \tau / \hbar}-e^{i 2 \cos (\hat{p} / \hbar) \tau / \hbar}}{e^{-i \epsilon W v(\hat{q}) \cos \phi \tau / \hbar}+e^{i 2 \cos (\hat{p} / \hbar) \tau / \hbar}}\right| n^{\prime}, j^{\prime}\right\rangle .
\end{aligned}
$$

This is the Maryland transformed eigenvalue equation including degrees of freedom of the monochromatic perturbation in the case of the AM.

\section{Acknowledgments}

This work is partly supported by Japanese people's tax via JPSJ KAKENHI 15H03701, and the authors would like to acknowledge them. They are also very grateful to Dr. T.Tsuji and Koike memorial house for using the facilities during this study.
[1] K.Ishii, Prog. Theor. Phys. Suppl. 53, 77(1973).

[2] L.M.Lifshiz, S.A.Gredeskul and L.A.Pastur, Introduction to the theory of Disordered Systems, (Wiley, New York,1988).

[3] G. Casati, B. V. Chirikov,F. M. Izraelev, J.Ford, Stochastic behavior of a quantum pendulum under a periodic perturbation (Springer-Verlag,Berlin,1979) ed. by G.Casati and J.Ford .pp334.

[4] G.Casati, I.Guarneri and D.L.Shepelyansky, Phys. Rev. Lett. 62, 345(1989).

[5] F.Borgonovi and D.L.Shepelyansky, Physica D109, 24 (1997).

[6] J.Chabe, G.Lemarie, B.Gremaud, D.Delande, and P.Szriftgiser, Phys. Rev. Lett. 101, 255702(2008).

[7] J. Wang and A. M. Garcia-Garcia, Phys. Rev. E 79,
036206(2009).

[8] G. Lemarie, H.Lignier, D.Delande, P.Szriftgiser, and J.C.Garreau, Phys. Rev. Lett. 105, 090601(2010).

[9] C. Tian, A. Altland, and M. Garst, Phys. Rev. Lett. 107, 074101(2011).

[10] M.Lopez, J.F.Clement, P.Szriftgiser, J.C.Garreau, and D.Delande, Phys. Rev. Lett. 108, 095701(2012).

[11] M. Lopez, J.-F. Clement, G. Lemarie, D. Delande, P. Szriftgiser, and J. C. Garreau, New J. Phys. 15, 065013(2013).

[12] H.S. Yamada, F. Matsui and K.S. Ikeda, Phys. Rev. E 92, 062908(2015). H. S. Yamada and K. S. Ikeda, in preparation

[13] D.L.Shepelyansky, Physica D 8, 208-222(1983).

[14] S. Fishman, D.R.Grempel, R.E.Prange, Phys. Rev. Lett. 
49, 509 (1982); D.R. Grempel, R.E. Prange and S. Fishman, Phys. Rev. A 29, 1639(1984); R.E. Prange, D.R. Grempel, and S. Fishman, Phys. Rev. B 29, 65006512(1984).

[15] I. Manai, J-F. Clement, R. Chicireanu, C. Hainaut, J. C. Garreau, P. Szriftgiser, and D. Delande, Phys. Rev. Lett. 115, 240603(2015).

[16] E. Doron and S. Fishman, Phys. Rev. Lett. 60, 867 (1988).

[17] B. Toloui and L. E. Ballentine, Quantum Localization for Two Coupled Kicked Rotors, arXiv:0903.4632.

[18] H. Yamada and K. S. Ikeda, Phys. Lett. A 328, 170 (2004).

[19] H. S.Yamada and K. S. Ikeda, Eur. Phys. J. B 87, 208 (2014).

[20] H.S.Yamada and K.S.Ikeda, Phys. Rev. E 82, 060102(R)(2010); Eur. Phys. J. B 85, 41(2012); Eur. Phys. J. B 85, 195(2012).
[21] D. Vollhardt and P. Wolfle, Phys. Rev. Lett. 45,482(1980). D. Vollhardt and P. Wolfle, Phys. Rev. B 22,4666(1980). D. Vollhardt and P. Wolfle, Phys. Rev. Lett. 48, 699(1982).

[22] P. Wolfle and D. Vollhardt, Int. J. Mod. Phys B 24, 1526(2010).

[23] The average over irrational random number is more efficient than the normal random number average, because $U\left(s \tau, \varphi_{0}\right)$ is a periodic with respect to $\varphi_{0}$.

[24] M. Kappus and F. Wegner, Z. Phys. B 45, 15 (1981).

[25] B. Derrida, E. J. Gardner, J. Physique 45, 1283 (1984).

[26] J.L. Pichard, J. Phys. C:Solid State Phys. 19, 1519(1986).

[27] J. Kroha, Physica A 167, 231(1990).

[28] S. Adachi, M. Toda and K. Ikeda, Phys. Rev. Lett. 61, 659 (1988); B.Gadway, J.Reeves, L.Krinner, D.Schneble Phys. Rev. Lett 110,190401 (2013) . 